\section{Monitoring Pc 4 photodynamic therapy in clinical trials of cutaneous T-cell lymphoma using noninvasive spectroscopy}

\author{
Tammy K. Lee, ${ }^{a}$ Elma D. Baron, ${ }^{b}$ and Thomas H. Foster ${ }^{a, *}$ \\ anniversity of Rochester, Institute of Optics and Department \\ of Imaging Sciences, Rochester, New York 14642 \\ ${ }^{\mathrm{b}}$ Case Western Reserve University, University Hospitals of \\ Cleveland, Department of Dermatology, Cleveland, \\ Ohio 44106
}

\begin{abstract}
Silicon phthalocyanine $\mathrm{PC}_{\mathrm{C}} 4$ photodynamic therapy (PC 4-PDT) has emerged as a potentially effective treatment for cutaneous T-cell lymphoma (CTCL). Noninvasive reflectance and fluorescence spectroscopy before, during, and after PDT may provide useful dose metrics and enable therapy to be tailored to individual lesions. We present the design and implementation of a portable bedside spectroscopy system for initial clinical trials of Pc 4-PDT of CTCL. Reflectance and fluorescence spectra were obtained from an early stage CTCL patient throughout the course of the PDT treatment. Preliminary patient data show a significant effect of Pc 4 on the tissue absorption, modest Pc 4 photobleaching, and heterogeneity of PC 4 within and between the lesions. () 2008 Society of Photo-Optical Instrumentation Engineers. [DOI: 10.1117/1.2939068]
\end{abstract}

Keywords: photodynamic therapy; spectroscopy; reflectance; fluorescence; Pc 4; clinical trials.

Paper 08077LR received Mar. 4, 2008; accepted for publication Apr. 29, 2008; published online Jun. 10, 2008.

Cutaneous T-cell lymphoma (CTCL) is a skin neoplasm characterized by epidermotropism of atypical T-cells. The mycosis fungoides (MF) variant of CTCL accounts for $50 \%$ of primary cutaneous lymphomas and manifests as patches and plaques in the early stages of the disease. There is no consistently effective treatment for CTCL free of adverse side effects. ${ }^{1}$ Photodynamic therapy (PDT) has emerged as potentially effective in trials using topical 5-aminolevulinic acid (ALA) ${ }^{2,3}$ More recently, a clinical trial of silicon phthalocyanine Pc 4-PDT at Case Western Reserve University (CWRU) has been initiated. Advantages of Pc 4 include intense absorption at longer wavelengths, relative photostability, and, with topical application, a shorter drug-light interval. In preclinical studies, T-cells were found to be more susceptible than keratinocytes to Pc 4-PDT-induced-apoptosis. ${ }^{4}$ Accordingly, patients with early stage MF malignancies were enrolled in a phase I clinical trial of topical Pc 4-PDT at CWRU. Results of the study are not yet published, but preliminary observations are encouraging with respect to safety and possible biological response. Although the mechanisms leading to apoptosis in

*Tel: 585-275-1347; E-mail: thomas.foster@rochester.edu

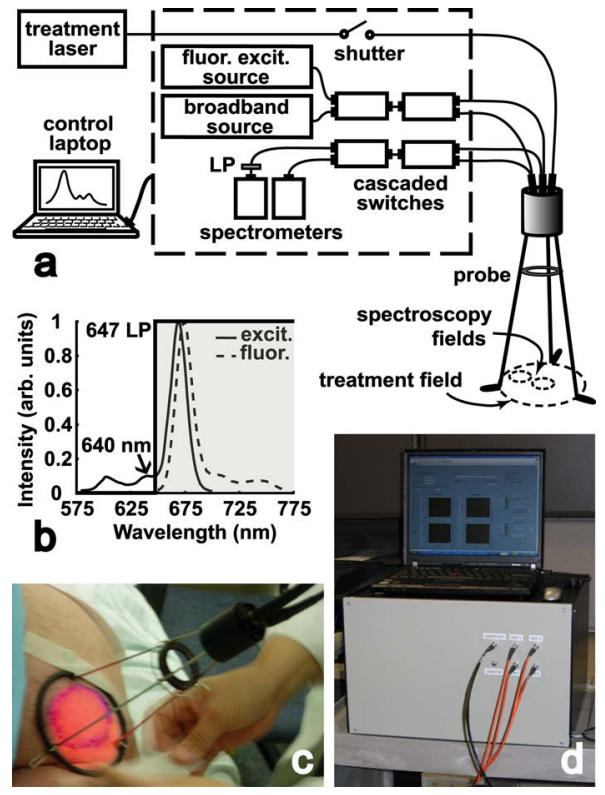

Fig. 1 (a) Schematic of the spectroscopy system. Within the housing are light sources, an optical routing system, and detectors. The probe accommodates spectroscopy from two $1-\mathrm{cm}$ spots within the $6-\mathrm{cm}$ treatment field. (b) Excitation and emission spectra of Pc 4. Fluorescence is excited at $640 \mathrm{~nm}$ allowing detection of the entire Pc 4 emission spectrum. A 647-nm long-pass filter rejects excitation light. (c) The custom probe stands $18 \mathrm{~cm}$ above the treatment surface on a tripod base. A diaphragm mounted on the tripod adjusts the treatment field size. (d) Photograph of the portable system and control laptop on a laboratory cart. Displayed on the laptop is the LabVIEW interface of the system control and data acquisition software.

response to in vitro Pc 4-PDT have been characterized, ${ }^{5}$ factors influencing dose deposition in patients have not previously been studied. Broadband diffuse reflectance and fluorescence spectroscopy before, during, and after PDT have the potential to inform tissue optical properties, sensitizer concentrations, and hemoglobin oxygen saturation $\left(\mathrm{SO}_{2}\right)$.

Thirty-four patients with early stage MF (TMN classification IA, IB, and IIA) were enrolled in the phase I clinical trial approved by the University Hospitals Institutional Review Board. The purpose of this study was to assess the safety of Pc 4 while escalating drug concentration $(0.01-0.1 \mathrm{mg} / \mathrm{mL})$ and light fluence $\left(50-200 \mathrm{~J} / \mathrm{cm}^{2}\right)$. First, $10 \mu \mathrm{L} / \mathrm{cm}^{2}$ of Pc 4 in $7: 3 \mathrm{EtOH} /$ propylene glycol is pipetted onto the treatment area $\left(5 \times 5 \mathrm{~cm}^{2} \max \right)$ followed by treatment with $675-\mathrm{nm}$ light $1 \mathrm{~h}$ later. To incorporate spectroscopy with PDT irradiation with minimal perturbation of the approved protocol, we designed and built a portable, easy to operate system, which was based on a device in clinical trials of ALA-PDT at Roswell Park Cancer Institute. ${ }^{6}$ Unlike that system, here we use commercial optical switches and a dedicated source for fluorescence excitation at a wavelength different than that used for PDT. This enables acquisition of the entire emission spectrum of Pc 4. Since various-sized lesions are treated in the CWRU trial, a probe with expanded capability to control the treatment field size was designed.

A schematic of the system is shown in Fig. 1(a). Integration with PDT necessitates only the input of the portable 


\section{JBO LETTERS}

675-nm 8-W treatment diode laser (AOC Medical Systems, South Plainfield, NJ) using an optical fiber. Enclosed within the housing are light sources for spectroscopy, an optical routing system, and components for detection. During acquisition of spectra, the PDT treatment light is interrupted by an inline transistor-transistor logic (TTL)-controlled shutter (Ocean Optics Inc., Dunedin, FL). In the light delivery arm are two sources; a TTL-shuttered 10-W tungsten halogen lamp (Avantes Inc., Broomfield, CO) for broadband reflectance and a 640-nm, 35-mW fiber-coupled laser diode (Optics Ltd., Ottawa, ON) for fluorescence excitation. Pc 4 fluorescence is excited at $640 \mathrm{~nm}$ in order to capture the entire emission spectrum [Fig. 1(b)]. The optical routing system consists of a pair of cascaded $2 \times 1$ fiber switches (Piezosystem Jena Inc., Hopedale, MA) in both delivery and detection arms. The fluorescence is long-pass filtered (Semrock Inc., Rochester, NY) to remove 640-nm excitation light. The two detectors are 16bit TE-cooled CCD spectrometers with 3-nm resolution (B\&W Tek Inc., Newark, DE).

The custom off-surface probe is positioned $18 \mathrm{~cm}$ above the treatment surface on a tripod base [Fig. 1(a) and 1(c)]. The treatment field size is adjusted with a diaphragm mounted on the tripod, permitting field sizes up to $6 \mathrm{~cm}$ in diameter. Terminated in the center of the probe is the treatment fiber, a $400-\mu \mathrm{m}$ core diameter microlens diffuser with a numerical aperture (NA) of 0.39 (Pioneer Optics Co. Inc., Bloomfield, $\mathrm{CT}$ ). Also terminated in the probe are two pairs of fibers for spectroscopic measurements of two interrogation locations within the treatment field; a fixed location in the center of the field and a second that is adjustable by means of a nylon ball bearing. Each pair of fibers consists of a light delivery fiber (600- $\mu \mathrm{m}$ core diameter; NA 0.22) and a detection fiber (400- $\mu \mathrm{m}$ core diameter fiber; NA 0.22). Both fibers are coupled to a 6-mm effective focal length lens resulting in a 1-cm-diameter interrogation spot. The size of the spot provides an acceptable signal-to-noise ratio while minimizing the duration of treatment interruptions. Differing angles of light delivery and detection reduce the amount of specular reflection detected. A set of spectroscopic measurements consists of interrogating the center and outer spots with broadband light, followed by $640-\mathrm{nm}$ fluorescence excitation. The integration time for each measurement is $1 \mathrm{~s}$; therefore, the duration of one set of measurements is $\sim 4 \mathrm{~s}$. Sets of spectroscopic measurements can be made before, during, and after PDT, after any number of delivered fluences.

System control and data acquisition is performed using a laptop, data acquisition card, and software programmed in LabVIEW (National Instruments, Austin, TX). The user interface allows the operator to enter patient information, take predrug measurements, and initiate PDT treatment with userdefined periodic spectroscopic measurements. Real-time preliminary data reduction is performed and displayed on the interface showing reflectance and fluorescence spectra from both interrogation spots. Spectra are corrected for background signals and system response generated using a National Institute of Standards and Technology (NIST)-traceable calibration lamp and diffuse reflectance standard (Ocean Optics Inc., Dunedin, FL). Further empirical corrections performed posttreatment address effects induced by absorption and scattering on the intrinsic fluorescence through division of the fluorescence spectra by the diffuse reflectance measured in the same

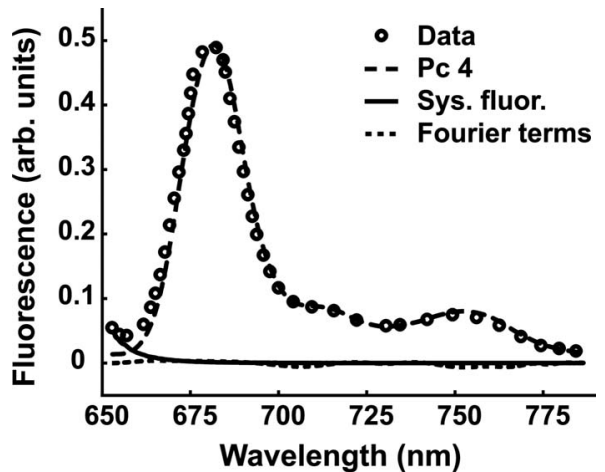

Fig. 2 Representative SVD fit to fluorescence data from a patient CTCL lesion (circles). At wavelengths shorter than $660 \mathrm{~nm}$ the signal is dominated by system fluorescence (solid line). At longer wavelengths, the spectrum is dominated by $\mathrm{PC}_{\mathrm{C}} 4$ fluorescence (dashed line). The Fourier series contribution (dotted line) never exceeds 2.2\% of the fit to the intrinsic fluorescence in the range $680-750 \mathrm{~nm}$, indicating a high-quality fit.

geometry and spectral window. ${ }^{7}$ In addition to Pc 4 emission, native system fluorophors contribute to the signal. Pc 4 fluorescence is extracted with a singular value decomposition (SVD) algorithm implemented in MATLAB (The MathWorks Inc., Natick, MA), which utilizes basis spectra for Pc 4 and system fluorescence and a Fourier series that captures contributions from unknown fluorophors or irradiation-induced fluorescence changes. ${ }^{6}$ A representative SVD fit to patient data is shown in Fig. 2. The signal below $660 \mathrm{~nm}$ is attributed to system fluorescence, whereas at longer wavelengths, the data are dominated by Pc 4 . A measure of the method's ability to return accurate estimates of Pc 4 fluorescence is that the amplitude of the Fourier terms never exceeds $2.2 \%$ of the fit to the intrinsic fluorescence in the range 680 to $750 \mathrm{~nm}$.

Three 6-cm diameter lesions in one patient were treated with a topically applied Pc 4 concentration of $0.1 \mathrm{mg} / \mathrm{mL}$. Immediately before irradiation, the lesion was cleaned with ethanol to remove excess Pc 4. The lesions were irradiated with $675-\mathrm{nm}$ light at a fluence rate of $100 \mathrm{~mW} / \mathrm{cm}^{2}$ for $200 \mathrm{~J} / \mathrm{cm}^{2}$. Twenty-nine sets of spectroscopic measurements were taken throughout the treatment. Twelve complete data sets were obtained: reflectance and fluorescence spectra from the center and outer regions from each of the three lesions. Four of the data sets were not included in the photobleaching analysis because of probe adjustments during treatment of the first of the lesions. Figures 3(a) and 3(b) show selected patient spectra from one location. The reflectance spectra [Fig. 3(a)] are normalized at $800 \mathrm{~nm}$, near an isosbestic point of hemoglobin. The presence of Pc 4 is indicated by decreased reflectance near $670 \mathrm{~nm}$, the main Pc 4 absorption peak. Before PDT $\left(0 \mathrm{~J} / \mathrm{cm}^{2}\right)$, the reflectance at $670 \mathrm{~nm}$ is equal to that at $\sim 629 \mathrm{~nm}$, which demonstrates the impact of Pc 4 on the tissue absorption under these treatment conditions. With increasing fluence, the $670-\mathrm{nm}$ reflectance progressively increases. The Pc 4 fluorescence extracted using SVD is shown in Fig. 3(b), normalized to the pre-PDT amplitude. The moderate decrease in Pc 4 fluorescence implies photobleaching, consistent with the reflectance spectra. To better examine this irradiation-induced effect, the Pc 4 fluorescence amplitude plotted against fluence is shown in Fig. 3(c). These data are 


\section{JBO LETTERS}

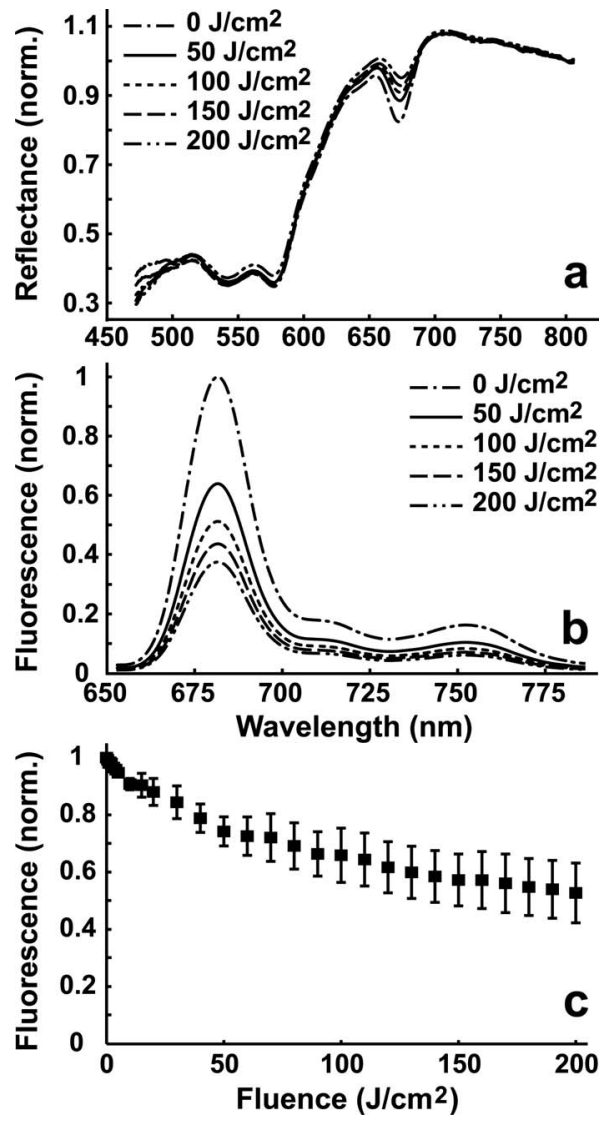

Fig. 3 (a) Representative in vivo reflectance curves at several fluences taken from one interrogation field. The curves are normalized at $800 \mathrm{~nm}$, near an isosbestic point of oxy- and deoxyhemoglobin. The decrease in reflectance near $670 \mathrm{~nm}$ is the result of Pc 4's strong absorption. With increasing fluence, the reflectance near $670 \mathrm{~nm}$ increases. (b) Corresponding Pc 4 fluorescence curves corrected for distortions caused by absorption and scattering. With increasing fluence, the amplitude of the Pc 4 fluorescence decreases. (c) Mean \pm SEM Pc 4 fluorescence amplitudes vs fluence from two interrogation spots in each of two lesions. Pc 4 undergoes modest photobleaching to 53\% of initial value after $200 \mathrm{~J} / \mathrm{cm}^{2}$.

means \pm standard error of the mean from measurements at two locations in each of two lesions. The fluorescence steadily decreases with irradiation reaching $\sim 53 \%$ of initial fluorescence levels at $200 \mathrm{~J} / \mathrm{cm}^{2}$. This photobleaching is quite modest in comparison to photobleaching rates of other photosensitizers. ${ }^{6}$

Heterogeneities in detected fluorescence were also observed. Figure 4 shows pre-PDT Pc 4 fluorescence levels from the center and outer regions in two lesions. Within lesion B, the fluorescence was $74 \%$ lower in the center vs outer region. Interlesion heterogeneity was also noted; the mean fluorescence of lesion A was $65 \%$ greater than that of B. Although Pc 4 was applied to both lesions at the same time, the sequence of lesion irradiation resulted in a longer drug-light interval for lesion B. The drug may have penetrated deeper into the tissue creating a lower concentration in the interrogation volume. Heterogeneities may also arise from variable distribution of Pc 4 on the surface, which could be secondary to properties of the vehicle.

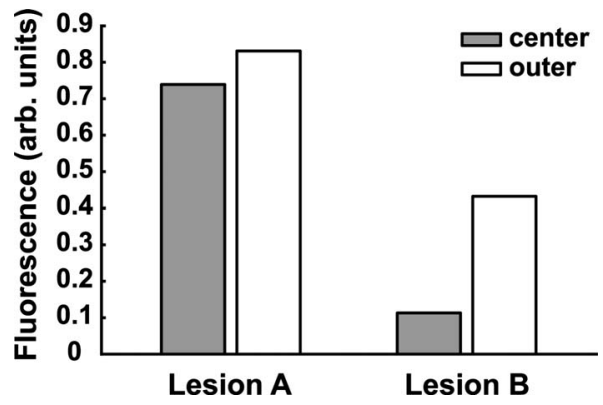

Fig. 4 Inter- and intralesion heterogeneities in Pc 4 fluorescence levels pre-PDT from two lesions. The mean fluorescence of Lesions A and $B$ different by $65 \%$. Within Lesions A and B, the PC 4 fluorescence between the center and outer regions varies by 13 and $74 \%$, respectively.

In summary, this system integrates spectroscopy with PDT for current and anticipated clinical trials of Pc 4-PDT. In preliminary patient data, the presence of Pc 4 significantly increased tissue absorption at the treatment wavelength, modest photobleaching was observed, and intra- and interlesion Pc 4 heterogeneities became apparent. These results emphasize the importance of optical dosimetry in the early stages of PDT clinical trials. Our research plans include the development of algorithms for extracting $\mathrm{SO}_{2}$ from off-surface measurements and characterization of the tissue volumes interrogated by spectroscopy using this probe.

\section{Acknowledgments}

We are grateful to Dr. Nancy Oleinick for helpful discussions on Pc 4-PDT and to Dr. Diana Santo Domingo, Dr. Valdir Colussi, and Jona Matevish for their clinical expertise. This work is supported by NIH/NCI Grant No. CA122093.

\section{References}

1. P. L. Zinzani, A. J. M. Ferreri, and L. Cerroni, "Mycosis fungoides," Crit. Rev. Oncol. Hematol. 65(2), 172-182 (2008).

2. K. Svanberg, T. Andersson, D. Killander, I. Wang, U. Stenram, S Andersson-Engels, R. Berg, J. Johansson, and S. Svanberg, "Photodynamic therapy of non-melanoma malignant tumours of the skin using topical $\delta$-amino levulinic acid sensitization and laser irradiation," Br. J. Dermatol. 130(6), 743-751 (1994).

3. A. Orenstein, J. Haik, J. Tamir, E. Winkler, H. Trau, Z. Malik, and G. Kostenich, "Photodynamic therapy of cutaneous lymphoma using 5-aminolevulinic acid topical application," Dermatol. Surg. 26(8), 765-770 (2000).

4. M. S. Ke, L.-Y. Xue, D. K. Feyes, K. Azizuddin, E. D. Baron, T. S. McCormick, H. Mukhtar, A. Panneerselvam, M. D. Schluchter, K. D. Cooper, N. L. Oleinick, and S. R. Stevens, "Apoptosis mechanisms related to increased sensitivity of Jurkat T-cells vs A431 epidermoid cells to photodynamic therapy with the phthalocyanine Pc 4," Photochem. Photobiol. 84(2), 407-414 (2008).

5. J. D. Miller, E. D. Baron, H. Scull, A. Hsia, J. C. Berlin, T. McCormick, V. Colussi, M. E. Kenney, K. D. Cooper, and N. L. Oleinick, "Photodynamic therapy with the phthalocyanine photosensitizer Pc 4: The case experience with preclinical mechanistic and early clinicaltranslational studies," Toxicol. Appl. Pharmacol. 224(3), 290-299 (2007).

6. W. J. Cottrell, A. R. Oseroff, and T. H. Foster, "Portable instrument that integrates irradiation with fluorescence and reflectance spectroscopies during clinical photodynamic therapy of cutaneous disease," Rev. Sci. Instrum. 77(6), 064302 (2006).

7. J. Wu, M. S. Feld, and R. P. Rava, "Analytical model for extracting intrinsic fluorescence in turbid media," Appl. Opt. 32(19), 3585-3595 (1993). 